\title{
Ueber Trimethylen;
}

\author{
von
}

\section{August Freund. ${ }^{1}$ )}

Es ist allgemein bekannt, von welchem Einflusse die Erkenntniss von Isomerien auf die Entwicklung unserer gegenwärtigen Ansichten über die Constitution organischer Verbindungen gewesen ist. Wenn demnach isomere Körper im Allgemeinen ein bedeutendes wissenschaftliches Interesse für sich in Anspruch nehmen, so ist leicht zu begreifen, dass solche von verhältnissmässig kleinem Moleculargewicht dies noch im höheren Grade thun müssen, weil dieselben einen leichteren Einblick in ihre innere Zusammensetzung gestatten.

Aus diesem Grunde ist denn auch unter Anderem die Darstellung eines isomeren Propylens mehrfach angestrebt worden, und wiewohl bei Weitem nicht alle Wege eingeschlagen wurden, welche zu dessen Auffindung führen konnten, so ist doch auf Grundlage einiger misslungener Versuche die etwas verfrühte Ansicht ausgesprochen worden, ein solches wäre überhaupt nicht existenzfähig, wenngleich die Kenntniss zweier isomerer Propylenbromüre die Existenzfähigkeit zweier Propylene mindestens als möglich erscheinen liess.

Unter den vielen Wegen, die zur Auffindung eines zweiten Propylens führen konnten, bot derjenige der Einwirkung von Natrium auf Trimethylenbromür die grösste Aussicht auf Erfolg. Nun ist allerdings die Einwirkung von Natrium auf Trimethylenbromür von $\mathrm{Reboul^{2 }}$ ) versucht worden, ohne demselben das erwünschte Resultat gebracht zu haben; demungeachtet war die Möglichkeit nicht ausgeschlossen, mittelst derselben Reaction unter modificirten Verhältnissen zum Ziele zu gelangen.

1) Vom Verf. aus den Sitzungsber. d. Wiener Acad. d. Wissensch. II. Abthlg., Juli-Heft, mitgetheilt.

$\left.{ }^{2}\right)$ Compt. rend. 78,1775 . 
Bei der Darstellung von normalem Butylalkohol aus Glycerin nach Fitz's Angabe, glücklicher Weise in den Besitz ansehnlicher Mengen von Trimethylenalkohol gelangt, und somit in der Lage, mir leicht vollständig reines Trimethylenbromür beschaffen zu können, glaubte ich die Gelegenheit, die erwähnte Einwirkung von Natrium auf Trimethylenbromür einem erneuten Studium unterziehen $\mathrm{zu}$ können, nicht ausser Acht lassen zu dürfen. Gleich beim ersten Versuch überzeugte ich mich, dass das Trimethylenbromür schon bei gewöhnlichem Luftdrucke leicht durch Natrium zerlegt wird, so dass ich nicht begreifen kann, warum Reboul die Einwirkung in geschlossenen Röhren vornahm.

$\mathrm{Zu}$ meinen Versuchen diente reines, bei $164,5^{\circ}-165,5^{\circ}$ siedendes, aus Trimethylenalkohol mittelst Bromwasserstoff dargestelltes Bromür. ${ }^{1}$ ) Die Einwirkung des Natriums auf das Bromür geschieht am besten in einem Kölbchen von circa $100 \mathrm{Ccm}$. Inhalt, an dessen etwa $10 \mathrm{Cm}$. langen Hals ein schief nach aufwärts gerichtetes, mit dem unteren Ende eines Liebig'schen Kühlers verbundenes Rohr angeschmolzen ist. In dem oberen Ende des Kühlers ist ein kleiner, mit wenig Alkohol gefülter Kugelwaschapparat befestigt, aus welchem das Gas in ein Gasometer geleitet wird. Nachdem zu dem Bromür einige Stückchen Natrium (etwa 0,5 Grm.) gebracht worden, wird das Kölbchen mit einem Stöpsel geschlossen, und hierauf nahe zum Siedepunkte des Bromürs erhitzt.

Alsbald beginnt eine energische Gasentwicklung, und ist es nöthig, zur Mässigung derselben die Lampe zu entfernen, und das Kölbchen erst dann wieder zu erwärmen, wenn die Gasentwicklung aufzuhören beginnt. Ist eine Portion Natrium in Bromnatrium verwandelt worden, so wird eine neue Quantität zugesetzt, und so fortgefahren, bis der Inhalt des Kölbchens in Folge des entstandenen Bromnatriums teigig geworden ist. $^{2}$ )

1) Monatshefte f. Chem. 1881, S. 639.

* Dies findet statt, nachdem anf $140 \mathrm{Grm}$. 'Trimethylenbromür etwa $\overline{5} \mathrm{Grm}$. Natrium eingewirkt haben. 
Es ist gut, während der Reaction, durch entsprechendes Bewegen des Kölbchens dafür zu sorgen, dass das Natrium mit dem Bromür in Berührung bleibe, was namentlich dann durchaus nöthig ist, wenn der Inhalt des Kölbchens teigig zu werden beginnt, da dann das Natrium im Bromnatrium eingehüllt und leicht ausser Contact mit dem Trimethylenbromür gesetzt wird. Ist der Inhalt des Kölbchens derart teigig geworden, dass das zugesetzte Natrium nicht mehr einwirken kann, so wird noch eine kurze Zeit erwärmt, dann erkalten gelassen, und, nachdem die Communication zwischen Gasometer und Kölbchen abgesperrt worden, dieses letztere entleert und von Neuem beschickt. ${ }^{1}$ ) Dazu kann entweder frisches Trimethylenbromür, oder dasjenige verwendet werden, welches man aus dem Produkt durch Absaugen mittelst einer Pumpe zurückgewinnen kann. ${ }^{2}$ )

Das in der angeführten Weise gewonnene Gas ist mit leuchtender Flamme brennbar und besitzt einen, dem gewöhnlichen Propylen und Butylen ähnlichen Geruch.

\section{Verhalten des Gases gegen Brom.}

Zur Constatirung der chemischen Eigenschaften des Gases wurde es zunächst durch Brom geleitet. Hierzu bediente ich mich eines Kugel-Apparates, welcher aus einem unter einem Winkel von etwa $60^{\prime \prime}$ gebogenen Glasrohre gefertigt war, dessen längerer (etwa $30 \mathrm{Cm}$. langer) Schenkel in fünf kleinere und eine dem Ende des Schenkels zunächst liegende grössere, der kürzere hingegen in eine einzige grössere Kugel ausgeblasen war. Nachdem der Kugel-Apparat

1) Zur Constatirung, dass das in dieser Weise erhaltene Gas nicht identisch ist mit dem gewöhnlichen Propylen, genügt übrigens eine einzige Operation mit etwa $140 \mathrm{Grm}$. Trimethylenbromür, wobei etwa 2 Lit. Gas erhalten werden, welche, wie weiter unten beschrieben werden soll, 5 Grm. normales Propyljodür zu liefern fähig sind.

$\left.{ }^{2}\right)$ Aus der teigigen Reactionsmasse konnten bei meinen Versuchen gegen $85 \mathrm{pCt}$. Trimethylenbromür zurückgewonnen werden, und zwar durch blosses Absangen ctwa 65 pCt, durch Extrahiren des auf dem Filter bleibenden Bromnatriums mit Aether (oder durch Lösen im Wasser) weitere 20 pCt.

Jourval f. prakt. Chemie [2] Bd. $\mathbf{2 6}$. 
mit Brom beschickt worden, wurde demselben eine solche Lage gegeben, dass der kürzere, mit einer Kugel versehene Schenkel senkrecht zu stehen kam. Nun wurde durch den Apparat das Gas bei der senkrecht stehenden Kugel hinein in einen mit Wasser gefüllten zweiten Gasometer geleitet.

Das Verhalten des Gases gegen Brom ist ein auffallend verschiedenes von jenem des gewöhnlichen Propylens, mit welchem ein Controlversuch angestellt wurde. Während nämlich das gewöhnliche Propylen leicht und vollständig bis zum Entfärben des Broms absorbirt wird, was namentlich bei etwas rascherem Hindurchleiten von merklicher Wärmeentwicklung begleitet ist, wirkt das aus 'Trimethylenbromür erhaltene Gas auf Brom anscheinend gar nicht ein. Anfänglich wurde der Versuch in der Weise gemacht, dass in den Kugel-Apparat wenig Brom (2-3 Ccm.) kam, und nun das Gas durch das Brom hindurch langsam, Blase für Blase, in ein zweites Gasometer geleitet wurde, Nach einmaligem Hindurchleiten hatte sich das Volum des Gases nur wenig vermindert, und nach dem Entleeren des Broms in ein Fläschchen und Durchschütteln mit einer Lösung von saurem schwefligsaurem Natron blieben nur wenige Tropfen einer schweren, mit Wasser nicht mischbaren Flüssigkeit zurück. Auch das Einstellen des Absorptionsapparates in directes Sonnenlicht hatte kaum besseren Erfolg; und erst dann, nachdem in den Kugel-Apparat eine grössere Menge Brom gegeben und das Gas mehrere Male hindurchgeleitet wurde, konnte eine für die Untersuchung hinreichende Menge Bromür gewonnen werden, wiewohl auch da noch eine merkliche Menge Gas unabsorbirt blieb.')

Das durch Einwirkung des Gases auf Brom gebildete

1) Eine Verbrennung zeigte, dass das rückständige Gas Kohlenstoff und Wasserstoff in demselben Verhältnisse enthielt, wie vor dem Hindurchleiten durch Brom. Auf 100 Thle. C wurden nämlich $17,7 \mathrm{H}$ gefunden - im frisch bereiteten Gase anf 100 Thle. C 18,1 H - für Propylen berechnen sich auf $100 \mathrm{C} 16,66 \mathrm{H}$, für Propan auf $100 \mathrm{C}$ 22,2 H. Die Vernuthung, dass bei der Einwirkung des Natriums auf Trimethylenbromür allenfalls unter Mitwirkung der Luftfeuchtigkeit Propan gebildet worden sei, fand sich demuach nicht bestätigt. 
Bromür erwies sich hinsichtlich der Zusammensetzung und der sonstigen Eigenschaften als identisch mit Trimethylenbromür. Nach entsprechendem Reinigen und Trocknen der Destillation unterworfen, zeigte das Thermometer, nachdem kaum einige Tropfen herüberdestillirt waren, $165^{\circ}$, und innerhalb dieser Temperatur und $180^{\circ}$ war alles überdestillirt. Bei wiederholter Destillation wog der unterhalb $165^{\circ}$ überdestillirte Antheil 1,3 Grm., jener zwischen $165^{\circ}-170^{\circ}$ $18 \mathrm{Grm}$, zwischen $170^{\circ}-180^{\circ} 7,5 \mathrm{Grm}$.

Der zwischen $165^{\circ}-170^{\circ}$ herübergegangene Antheil wurde für die Analyse und Bestimmung des specifischen Gewichts verwendet, welch letzteres $=1,978$ bei $19^{\circ}$ gegen Wasser von $4^{0}$ gefunden wurde.

0,422 Grm. gaben 0,7784 Grm. Ag Br, entsprechend 78,46 pCt. Br.

$0,7402 \mathrm{Grm}$. gaben im Sauerstoffstrom bei vorgelegtem Silber, verbrannt, 0,4706 Grm. $\mathrm{CO}_{2}$, and 0,1974 Grm. Wasser.

$\begin{array}{lcc} & \text { Gefunden. } & \text { Berechnet. } \\ \mathrm{C} & 17,34 & 17,82 \\ \mathrm{H} & 2,96 & 2,97 \\ \mathrm{Br} & 78,46 & 79,19\end{array}$

Verhalten des Gases gegen Jodwasserstoff.

Liefert schon das Verhalten des aus Trimethylenbromür mittelst Natrium erhaltenen Propylens gegenüber Brom einen hinreichenden Beweis für die Nichtidentität desselben mit dem gewöhnlichen Propylen, so ist dies hinsichtlich seines Verhaltens gegenüber Jodwasserstoff noch in weit höherem Grade der Fall.

Das Verhalten des Gases gegenüber Brom liess erwarten, dass dasselbe dem Jodwasserstoff gegenüber sich nun vollends reactionsunfähig erweisen werde. Der Versuch zeigte jedoch das Gegentheil, und lieferte einen Beweis mehr, wie trügerisch Analogieschlüsse sein können. Es zeigte sich nämlich, dass das Trimethylen, wenn nicht leichter, so doch jedenfalls nicht schwerer von rauchender Jodwasserstoffsäure bei gewöhnlicher Temperatur absorbirt wird, wie das gewöhnliche Propylen, mit welchem auch hier ein Controll. versuch angestellt wurde. 
Als Absorptionsapparat diente ein mit seitlich angeblasenem Rohr versehenes Kölbchen, in dessen Hals ein $20 \mathrm{Cm}$. langes, $2 \mathrm{Cm}$. breites, mit Glasperlen gefülltes und beiderseits eng ausgezogenes Rohr mittelst eines Korkes derart befestigt war, dass das untere Ende des Rohres bis nahe am Boden des Kölbchens mündete; das obere rechtwinkelig gebogene Ende des Rohrs war mit drei kleinen Kugelapparaten in Verbindung gesetzt, von denen der erste Wasser, der zweite 3--4 Ccm. Alkohol, der dritte wieder Wasser enthielt. Dieser Letztere stand mit einem mit Wasser gefüllten Gasometer in Verbindung. Nachdem in das Kölbchen eine entsprechende Menge (etwa $20 \mathrm{Ccm}$., höchst concentrirter Jodwasserstoffsäure eingefüllt worden) wurde die seitliche Oeffnung des Kölbchens mit dem das Gras enthaltenden Gasometer in Verbindung gesetzt, nachdem noch zuvor ein kleines mit $\mathrm{CaCl}_{2}$ gefülltes TrockenRöhrchen dazwischen eingeschaltet worden. Durch den Druck des Gases wurde die Jodwasserstoffsäure in das mit Glasperlen gefüllte Rohr senkrecht in die Höhe getrieben, wodurch einerseits ein kleiner Druck im Apparate hergestellt, und andererseits dem Gase die Möglichkeit geboten wurde, mit der Jodwasserstoffsäıre in möglichst innige Berührung $\mathrm{zu}$ treten.

Nachdem das Gas durch den Absorptionsapparat hindurch in den andern Gasometer getrieben worden, wurde die Lage der Gasometer gewechselt, worauf abermals das Gas aus einem Gasometer in den andern geleitet wurde.

Dies wurde so oft wiederholt, his das aus dem $\mathrm{Ab}$ sorptionsapparat austretende Gas sich als nicht mehr brennbar erwies. ${ }^{1}$ ) Während der ganzen Operation, welche etwa ein achtmaliges Hindurchleiten des Gases durch den Ab-

1) Schon das ursprünglich zur Verwendung gebrachte Gas musste eine gewisse Quantität atmosphürischer Luft enthalten. Wollte man nämlich nicht viel an Gas verlieren, so musste gleich zu Anfang, elie noch sämmtliche aus dem Apparat stammende Luft verdringt war, das Gas in den Gasometer geleitet werden. Während der Absorption kam auch noch etwas Luft hinzu, welche selbstverstindlich zurückbleiben musste. 
sorptionsapparat forderte, wurde die Jodwasserstoffsäure zweimal durch frische ersetzt. Nach beendeter Einwirkung wurde der Inhalt des Kölbchens mit Wasser verdünnt, mit etwas schwefeliger Säure entfärbt und solange destillirt, bis noch Oeltropfen in die Vorlage übergingen. Bei der Destillation wurde zwischen Kölbchen und Kühler das mit Glasperlen gefüllte Rohr eingeschaltet, um das den Glasperlen adhärirende Jodür nicht zu verlieren. Aus dem mit Alkohol gefüllten Kugel-Apparate konnte durch Wasser 'ebenfalls eine kleine Menge Jodürs abgeschieden werden.

Im Ganzen erhielt ich bei Verarbeitung von etwa 4 Lit. Gases $10 \mathrm{Grm}$. an Jodür, welches fast vollständig, d. i. $8,5 \mathrm{Grm}$. zwischen $101,5^{0}-103^{01}$ ) beim Drucke von 736,5 Mm. herüberdestillirte (im Kölbchen blieben 0,8 Grm.) und bei $24^{\circ}$ ein specifisches Gewicht $=1,7187$ gegen Wasser von $4^{\circ}$ besass.

0,4396 Grm. dieses Jodürs gaben 0,6064 Grm. AgJ, entsprechend $74,54 \mathrm{pCt}$. Jod. Die Rechnung verlangt für $\mathrm{C}_{3} \mathrm{H}_{7} \mathrm{~J} 74,7 \mathrm{pCt}$. J.

Dass dieses Jodür normales Propyljodür ist, dafür spricht ausser dem Siedepunkte auch noch der Umstand, dass dasselbe die von V. Meyer entdeckte für primäre Alkohole charakteristische Nitrolsäure-Reaction in prägnantester Weise gab.

Es wäre somit ausser $Z$ weifel gestellt, dass zwei Kohlenwasserstoffe von der empirischen Formel $\mathrm{C}_{3} \mathrm{H}_{6}$ existenzfäbig sind, und es handelt sich nur noch darum, welche Constitution denselben zuerkannt werden soll.

Bei der Leichtigkeit, man könnte sagen Leichtfertigkeit, mit welcher gegenwärtig über die Constitution höchst complicirter Verbindungen abgeurtheilt wird, sollte man meinen, dass bei so einfachen Körpern, wie dies die beiden Propylene sind, gar kein Zweifel darüber herrschen könne, welche Constitution jedem derselben zukomme.

Und in der That, unter Voraussetzung, dass dem ge-

1) Bei einem Controlversuch in äbnlicher Weise aus gewöhnlichem Propylen dargestelltes Propyljodür destillirte vollständig zwischen $87^{\circ}$ bis $92,5^{\circ}$ herüber. 
wöhnlichen Propylen die in der Formel: $\mathrm{CH}_{3}-\mathrm{CH}=\mathrm{CH}_{2}$ zum Ausdruck gebrachte Constitution zukömmt, würde für das neue Propylen (einer zweiten Voraussetzung nach, nämlich dass in beiden Kohlenwasserstoffen vierwerthiger Kohlenstoff functionire) nur die Formel:<smiles>CCCC</smiles>

zulässig sein. Giebt man jedoch $\mathrm{zu}$, dass eines der drei Kohlenstoffatome als zweiwerthig functionire, dann wären die Structur-Formeln:

I.<smiles>C=C(C)C</smiles>

II.<smiles>CCC</smiles>

III.

IV.

möglich, und die Entscheidung, welche von ihnen dem gewöhnlichen, und welche dem neuen Propylen zuzuschreiben sei, ist dann weniger leicht.

Der Umstand, dass das gewöhnliche Propylen unter Anderen, nach Friedel und Ladenburgi ${ }^{1}$ ), aus Methylchloracetol durch Einwirkung von Natrium entsteht, würde für die erste Formel sprechen, wonach dasselbe als zweifach methylirtes Methylen aufzufassen wäre. Damit stünde auch im vollständigen Einklange die Bildung von secundärem Propyljodür aus gewöhnlichen Propylen und HJ. Der Umstand hingegen, dass aus gewöhnlichem Propylen durch Vereinigung mit Chlor ein von dem Methylchloracetol verschiedenes Chlorür entsteht (und ebenso durch Vereinigung mit Brom ein von dem Methylbromacetol verschiedenes Bromür), so wie die Umwandelbarkeit des Propylenbromürs in Glycol und gewöhnliche Milchsäure, sprechen wiederum zu Gunsten der Formel III, mit welcher ebenso gut die Bildung von secundärem Propyljodür in Einklang gebracht werden kann, wenn man die Regel gelten lässt, dass bei-Anlagerungen. von HJ, das Jod an das mit der geringsten Menge Wasserstoffs verbundene Kohlenstoffatom trete.

1) Bull. soc. chem. [2] 8, 146, u. Zeitschr. f. Chem. 1868, S. 47. 
Freund: Ueber Trimethylen.

$\mathrm{Ob}$ in den beiden Propylenen überhaupt ein Kohlenstoffatom als zweiwerthig functionirend angenommen werden kann, darüber schien mir das Verhalten der beiden Propyljodüre gegenüber Kalihydrat Aufschluss geben zu können.

Die Lostrennung der Elemente des Jodwasserstoffs kann nämlich entweder in der Weise stattfinden, dass - wie dies allgemein angenommen wird - das Jodatom von einem, und das Wasserstoffatom von einem benachbarten Kohlenstoffatome entnommen wird, in welchem Falle aus beiden Jodüren ein und dasselbe Propylen entstehen müsste im Sinne der Gleichungen:

$$
\begin{aligned}
& \mathrm{CH}_{3}-\mathrm{CH} \mathrm{H}-\mathrm{CH}_{2} \mathrm{~J}=\mathrm{CH}_{3}-\mathrm{CH}=\mathrm{CH}_{2}+\mathrm{HJ} \\
& \mathrm{CH}_{3}-\mathrm{CH} \mathrm{J}-\mathrm{CH}_{2} \mathrm{H}=\mathrm{CH}_{3}-\mathrm{CH}=\mathrm{CH}_{2}+\mathrm{HJ}
\end{aligned}
$$

oder aber, es könnte Jod und Wasserstoff von ein und demselben Kohlenstoffatome sich lostrennen, in welchem Falle zwei verschiedene Propylene entstehen müssten, wie aus den Gleichungen:

$$
\begin{aligned}
& \mathrm{CH}_{3}-\mathrm{CH}_{3}-\mathrm{CH} \quad \mathrm{HJ}=\mathrm{CH}_{3}-\mathrm{CH}_{2}-\stackrel{\mathrm{C}}{\mathrm{CH}}+\mathrm{HJ} \\
& \mathrm{CH}_{3}-\mathrm{C}: \mathrm{HJ}-\mathrm{CH}_{3}=\mathrm{CH}_{3}-\mathrm{C}-\mathrm{CH}_{3}+\mathrm{HJ}
\end{aligned}
$$

$\mathrm{zu}$ ersehen ist.

Endlich wäre noch der Fall denkbar, dass die beiden Jodüre gegen Kalihydrat, hinsichtlich Abspaltung von Jodwasserstoff, sich verschieden verhalten, in welch letzterem Falle jedoch ebenfalls zwei verschiedene Propylene resultiren müssten nach den Gleichungen:

$$
\begin{aligned}
\mathrm{CH}_{3}-\mathrm{CH}_{2}-\mathrm{CH} \mathrm{HJ} & =\mathrm{CH}_{3}-\mathrm{CH}_{2}-\mathrm{CH}+\mathrm{HJ} \\
\mathrm{CH}_{3}-\mathrm{CH} \mathrm{J}-\mathrm{CH}_{2} \mathrm{H} & =\mathrm{CH}_{3}-\mathrm{CH}=\mathrm{CH}_{2}+\mathrm{HJ}
\end{aligned}
$$

oder

$$
\begin{aligned}
\mathrm{CH}_{3}-\mathrm{CH} \mathrm{H}-\mathrm{CH}_{2} \mathrm{~J} & =\mathrm{CH}_{3}-\mathrm{CH}=\mathrm{CH}_{2}+\mathrm{HJ} \\
\mathrm{CH}_{3}-\mathrm{CHJ}-\mathrm{CH}_{3} & =\mathrm{CH}_{3}-\mathrm{C}-\mathrm{CH}_{3}+\mathrm{HJ}
\end{aligned}
$$

Hinsichtlich des Verhaltens der beiden Propyljodüre 
gegenüber Kalihydrat fand ich zwar Angaben ${ }^{1}$ ), wonach zu schliessen wäre, das aus beiden ein und dasselbe, und zwar das gewöhnliche Propylen entsteht, demungeachtet hielt ich es nicht für überflüssig, mich hiervon persönlich zu überzeugen. Ich habe deshalb einerseits normales, aus Gährungspropylalkohol mittelst Jodwasserstoff dargestelltes Propyljodür, andererseits secundäres, aus (durch Reduction von reinem Aceton mittelst Natrium gewonnenem) Isopropylalkohol in gleicher Weise bereitetes Jodür auf Kalihydrat in wässriger und alkoholischer Lösung einwirken lassen. Im ersteren Falle fand weder beim primären noch beim secundären Jodür Einwirkung statt, in alkoholischer Lösung aber wurde, ebenso aus einem, wie auch dem andern Jodür ein und dasselbe Propylen, und zwar das gewöhnliche gewonnen, welches von Brom leicht und vollständig absorbirt wurde, und ein unter dem Drucke von $726 \mathrm{Mm}$. bei $140,5^{\circ}-$ $141,5^{\circ}$ siedendes Propylenbromür lieferte, dessen specifisches Grewicht $=1,9267$ bei $19,5^{\circ}$ (gegen Wasser von derselben Temperatur) gefunden wurde.

Gegenüber diesem Verhalten der beiden Propyljodüre kann demnach kein $Z$ weifel mehr darüber herrschen, dass die für das gewöhnliche Propylen allgemein gebrauchte, von Erlenmeyer herrührende Formel: $\mathrm{CH}_{3} \mathrm{CH}=\mathrm{CH}_{2}$ die richtige ist. Für das neue Propylen würde demnach nur zwischen den Formeln:

$$
\mathrm{CH}_{3}-\mathrm{CH}_{2}-\mathrm{CH} \text { und } \mathrm{H}_{2} \mathrm{C}<_{\mathrm{CH}_{2}}^{\mathrm{CH}_{2}}
$$

die Wahl zu treffen sein.

Gegen die erstere Formel spricht der Umstand, dass aus dem neuen Propylen durch Vereinigung mit Brom, Trimethylenbromür entsteht, über dessen Constitution gegenüber der Uimwandlung desselben in Trimetliylenalkohol und der Oxydirbarkeit dieses letzteren zu Aethylenmilchsäure,

1) Die eine bezüglich des Isopropyljodürs von Erlenmeyer, Ann. Chern. Pharm. 133, 228; die andere bezüglich des norm. Propyljodürs von Chancel, Ann. Chem. Pharm. 151, 305. 
sowie nach der Beobachtung, dass der Trimethylenalkohol in $\gamma$-Oxybuttersäure, wovon an einem anderen Orte die Rede sein soll, vermittelst einfacher Reactionen überführbar ist, kein $Z_{w}$ eifel herrschen kann. Von einem nach der Formel:

$\mathrm{CH}_{3}-\mathrm{CH}_{2}-\mathrm{CH}$ constituirten Propylen wäre übrigens zu erwarten, dass es durch Vereinigung mit Chlor ein Chlorür von der Formel: $\mathrm{CH}_{3}-\mathrm{CH}_{2}-\mathrm{CHCl}_{2}$, d. i. das bekannte Propylidenchlorür gäbe. Nun ist zwar allerdings das Gegentheil hiervon nicht experimentell bewiesen worden, - dem Verhalten des neuen Propylens gegenüber Brom, und der Ueberführbarkeit des dabei resultirenden Trimethylenbromürs in das bei $119,5^{\circ}$ siedende Trimethylenchlorür nach, kann es jedoch kaum zweifelhaft sein, dass das neue Propylen sich mit Chlor nicht zu Propylidenchlorür, sondern zu dem entsprechenden Trimethylenchlorür verbinden werde. Es bliebe sonach für das neue Propylen, nur die Formel:<smiles>C1CC1</smiles>

übrig, und der Name "Trimethylen" gerechtfertigt selbst für den Fall, dass man in demselben sogenannte freie Valenzen annehmen wollte, wofür aber dem Verhalten des Trimethylens nach vorläufig kein Grund vorhanden ist.

Schliesslich sei mir gestattet, Herrn Joseph Frühling, welcher so freundlich war, eine grössere Parthie Glyceringährungsrückstände auf Trimethylenalkohol aufzuarbeiten, und auch sonst bei der vorliegenden Untersuchung mir behülffich gewesen ist, hiermit meinen besten Dank auszusprechen. 